\title{
Future Experimental Programs
}

\section{Hitoshi Murayama}

Department of Physics, University of California, Berkeley, California 94720, USA

Theoretical Physics Group, Lawrence Berkeley National Laboratory, Berkeley, California 94720, USA

Kavli Institute for the Physics and Mathematics of the Universe (WPI), Todai

Institutes for Advanced Study, University of Tokyo, Kashiwa 277-8583, Japan

E-mail: hitoshi@berkeley.edu, murayama@lbl.gov, hitoshi.murayama@ipmu.jp

\begin{abstract}
I was asked to discuss future experimental programs even though I'm a theorist. As a result, I present my own personal views on where the field is, and where it is going, based on what I myself have been working on. In particular, I discuss why we need expeditions into high energies to find clues to where the relevant energy scale is for dark matter, baryon asymmetry, and neutrino mass. I also argue that the next energy frontier machine should be justified on the basis of what we know, namely the mass of the Higgs boson, so that we will learn what energy we should aim at once we nail the Higgs sector. Finally I make remarks on dark energy.
\end{abstract}




\section{Introduction}

The discovery of a "Higgs-like particle" on July 4, 2012 was a truly historic moment in the history of science [1, 2]. Many of us in the United States watched the seminar at CERN over webcast in the midnight hours. Given that it was announced on the Independence Day of the United States, we celebrated the Higgsdependence Day in the early morning.

So far, what we've seen looks minimal. Later, a CERN announcement made in March 2013 said it is a Higgs boson. Indeed, the newly discovered particle looks very much like the Standard Model Higgs boson. We've been after this particle ever since 1933 when Fermi wrote his theory of nuclear beta decay. There, he introduced a constant $G \approx 10^{-5} m_{p}^{-2}$ which we now call the Fermi constant $G_{F}$. It corresponds to the energy scale $G_{F}^{-1 / 2} \approx 300 \mathrm{GeV}$, and we learned from him that something is going on at this energy scale. It took a whopping eighty years to come to the point where we now have a UV-complete theory of strong, weak, and electromagnetic forces with all of the parameters measured. In fact, it is a renormalizable and consistent theory that may be valid all the way up to the Planck scale. Coincidentally, even cosmology looks minimal given the Planck data [3], which suggests a minimal single-field inflation. Maybe the year 2013 will be remembered in history as the year of elementary scalars.

Despite this achievement, or rather because of it, there is a building anxiety in the community. How come we don't see anything else? Will the progress stop? There is no sign of physics beyond the Standard Model in the LHC data. For a typical search for supersymmetric particles, for example, squarks and gluinos are excluded up to $1.3 \mathrm{TeV}$ or so. On the other hand, the conventional arguments based on the naturalness concept suggested that we should have new particles that stabilize the electroweak scale below TeV. It appears that "natural and simple" models have been excluded (Fig. 1). Then we have two directions to go in: the less natural, namely fine-tuned, or the less simple, namely contrived. At the same time, theorists are trying to come up with models that can evade the current experimental limits, pushing back on this problem. See Appendix A for my own recent attempts.

I have to point out, however, that certain levels of fine-tuning do occur in nature. All examples I'm aware of, with the glaring exception of the cosmological constant, are at most at the level of a few per-mille. The current LHC limit has not quite reached that level; the next runs at 13-14 TeV may well reveal new physics as we hoped for. I will come back to this question later in this talk.

In any case, it is true that experimental limits have started to haunt theorists. Theorists used to complain that the experiments had a hard time keeping up with their new ideas. Now the tide has reversed. Theorists are being threatened by new data. I believe this is quite a healthy field!

Nonetheless, having a fully UV-complete theory of the Minimal Standard Model, now supported by the new particle that has been discovered, makes us ask the following question: 


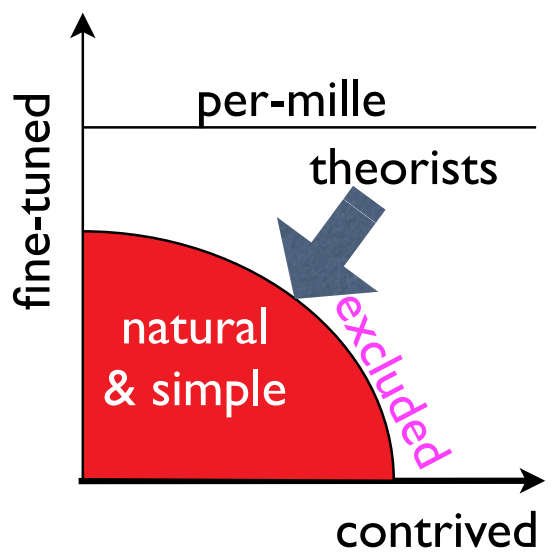

Figure 1. Schematic constraints on space of theories.

\section{Is particle physics over?}

On this question, fortunately, the answer is a resounding no. Since 1998, we have discovered five pieces of empirical evidence for physics beyond the Standard Model thanks to tremendous progress in experiments.

First, non-baryonic dark matter. Even though dark matter had been discussed since 1930's by Fritz Zwicky, it was not clear whether dark matter would be dark astronomical objects or hidden baryons. This issue was completely settled in 2003. The search for dark astronomical objects (MACHOs = Massive Compact Halo Objects) excludes the possibility that Galactic halo consists solely of MACHOs between about $\times 10^{-7} M_{\odot}$ and $10 M_{\odot}$ [4]. On the other hand, the power spectrum in the Cosmic Microwave Background (CMB) anisotropy by WMAP (Wilkinson Microwave Anisotropy Probe) excludes the baryonic dark matter completely as a discrepancy between the overall matter density $\Omega_{M} h^{2}=0.14 \pm 0.02$ and the baryon density $\Omega_{b} h^{2}=0.024 \pm 0.001$ [5]. We are learning what dark matter is not, but not what it is. In fact, we know so little that the only model-independent lower limit on the dark matter mass comes from the requirement that its "Bohr radius" in the gravitational potential must fit within the galactic scale [6]. Combined with the MACHO search, we managed to narrow down its mass from $10^{-31} \mathrm{GeV}$ to $10^{50} \mathrm{GeV}$, i.e., to within 81 orders of magnitude. Zwicky must be happy to see our progress! Thus, we need to keep our minds very open about the nature of dark matter.

The flavor oscillation of neutrinos, and hence their finite masses, is not a part of the Minimal Standard Model either, arguably the first established physics beyond the Standard Model in 1998 [7], revealing the mixing angle $\theta_{23}$. Later on, the oscillation (or rather lack of it as a result of the matter effect) of solar neutrinos [8] and oscillation of reactor neutrinos [9] pointed to the same parameter set (and the angle $\theta_{12}$ ) in 2002 resolving a puzzle that goes back half a century. The final mixing angle $\theta_{13}$ was discovered in 2012 [10]. Some people think it is only a minor extension of the Standard Model, but it should be emphasized that we don't yet know how it should be extended. 
The accelerated expansion of the Universe came as a big surprise to all of us [11, 12]. Its cause is now called dark energy, even though we are very far away from understanding what it is. It may be cosmological constant, due to a miraculous cancellation between quantum fluctuation of the vacuum and a classical constant energy density for 120 digits. It may be some dynamical substance called quintessence. Either way, it is very difficult to understand its overall amount.

At the same time, the observed apparently acausal density fluctuations in the CMB cannot be explained by the Standard Model. The CMB photons that came from one end of the Universe have just reached us; they seem to be correlated with the CMB photons that came from the other end, when they have had no chance to meet and set up their temperatures. This is what I mean by acausal. The best explanation is that they were in fact in causal contact early on because the entire visible Universe was much smaller than a nucleus; it was later stretched to a macroscopic size by an exponential expansion called inflation. The latest Planck data strongly supports this idea [3]. We normally assume that it was caused by a scalar field called the inflaton rolling slowly down the hill, but we don't know what it is, nor how it couples to the Standard Model particles.

Finally, once we accept the inflationary paradigm, the cosmic baryon asymmetry $\eta_{b}=n_{b} / n_{\gamma} \approx 5 \times 10^{-10}$ cannot be assumed to be the initial condition of the Universe. This is because the enormous exponential expansion (normally assumed to be more than $e^{60}$ ) wipes out any pre-existing asymmetry. This implies that the baryon asymmetry needs to be created after the inflation by a microphysical process. On the other hand, the CP violation in the Standard Model is now known to be incapable of producing enough baryon asymmetry. This is because that we now have understood the known CP violating phenomena by the Kobayashi-Maskawa theory thanks to the $B$-factory experiments starting in 2001 [13, 14]. This means that the Standard Model cannot generate the baryon asymmetry larger than the Jarlskog invariant $J=\Im m\left(\operatorname{Tr}\left[Y_{u}^{\dagger} Y_{u}, Y_{d}^{\dagger} Y_{d}\right]\right) \approx 10^{-20}$ [15], further suppressed by small efficiencies or powers of coupling constants in known mechanisms.

So, it is clear that particle physics is far from over. There are at least five important pieces of data that are crying out to be explained and understood. The catch is that we don't know the energy scale of physics relevant to these mysteries. Right now we are on fishing expeditions. In particular, we are and will continue to be looking for new phenomena and new sources of CP violation in the quark sector (LHCb, SuperKEKB, rare kaon decays), lepton sector (neutrino oscillations, neutrinoless double-beta decay, and electric dipole moments), and their combination (proton decay). We try to cast a wide net, hoping to catch any interesting fish, so that we learn where the next important energy scale is. In a sense, this is what Fermi succeeded in doing; by observing rare phenomena of nuclear $\beta$-decays, which violate conservation law of neutron and proton numbers that all other known forces respect, they were caught in the net and we learned about the Fermi scale.

Whatever the next energy scale beyond the Standard Model is, it plays the role 


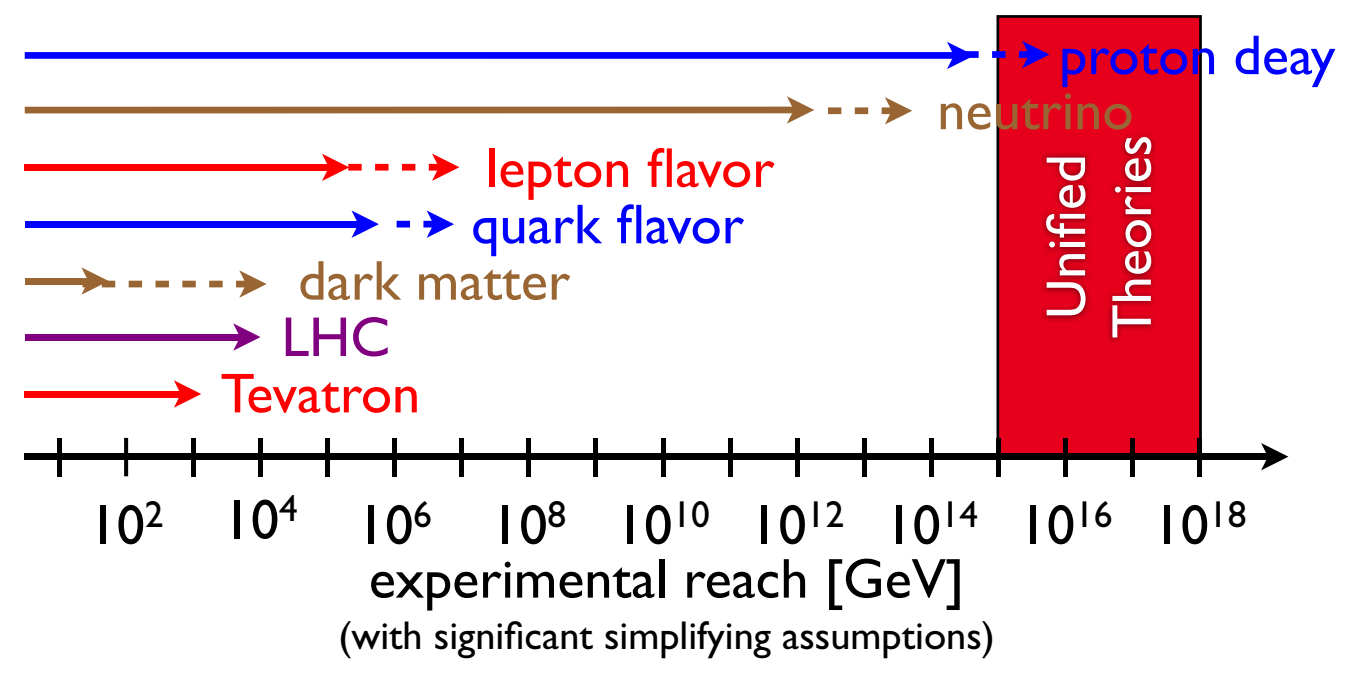

Figure 2. Approximate energy reach for expeditions. Solid arrows indicate the current reach, while the dashed arrows anticipated improvements by proposed experiments [16].

of the UV cutoff of the Standard Model as a low-energy effective field theory. And the effects of the UV cutoff can be parametrized by higher dimension operators power suppressed by $\Lambda_{U V}$ added to the Standard Model,

$$
\mathcal{L}=\mathcal{L}_{S M}+\frac{1}{\Lambda_{U V}} \mathcal{L}_{5}+\frac{1}{\Lambda_{U V}^{2}} \mathcal{L}_{6}+\cdots
$$

We need to first look at the Standard Model Lagrangian $\mathcal{L}_{S M}$ which has the structure as shown on T-shirts from CERN designed by John Ellis,

$$
\begin{aligned}
\mathcal{L}_{S M}= & -\frac{1}{g^{2}} F_{\mu \nu}^{2}+\bar{\psi} i \not D \psi+\left|D_{\mu} H\right|^{2}-y \bar{\psi} \psi H+\frac{\theta}{64 \pi^{2}} F \tilde{F}-\lambda\left(H^{\dagger} H\right)^{2} \\
& +\mu^{2} H^{\dagger} H-\Lambda_{C C} .
\end{aligned}
$$

The first line here has only dimensionless parameters and is only logarithmically sensitive to the physics at $\Lambda_{U V}$. On the other hand, the last line has two parameters $\mu^{2}$ (mass dimension 2) and $\Lambda_{C C}$ (4) and remember physics at $\Lambda_{U V}$, the origin of the naturalness problems we will come back to later.

On the other hand, the power-suppressed operators come in a great variety. For instance, those suppressed by two powers can be

$$
\mathcal{L}_{6}=Q Q Q L, \bar{L} \sigma^{\mu \nu} W_{\mu \nu} H l, \epsilon_{a b c} W_{\nu}^{a \mu} W_{\lambda}^{b \nu} W_{\mu}^{c \lambda},\left(H^{\dagger} D_{\mu} H\right)^{2}, B_{\mu \nu} H^{\dagger} W^{\mu \nu} H, \cdots
$$

They may be seen in proton decay, $g_{\mu}-2$, triple gauge boson vertex, $T$ and $S$-parameters in the precision EW observables, respectively.

It is interesting to note that there is actually only one-type of operator we can write suppressed by a single power,

$$
\mathcal{L}_{5}=(L H)(L H) .
$$


After substituting the expectation values for the Higgs field, it is nothing but the Majorana neutrino mass operator,

$$
\frac{1}{\Lambda_{U V}} \mathcal{L}_{5}=\frac{v^{2}}{\Lambda_{U V}} \nu \nu
$$

In other words, the neutrino mass can be viewed as the leading order effect of the physics beyond the Standard Model!

The neutrino mass is actually a tiny effect. Any kinematic effect is suppressed by $m_{\nu}^{2} / E_{\nu}^{2} \approx(0.1 \mathrm{eV} / \mathrm{GeV})^{2} \approx 10^{-20}$ ! Normally we don't think we can be sensitive to such a small number in experiments. However, there is one known technique that is sensitive to very small numbers: interferometry, like in the Michaelson-Morley experiment. For this to be possible, there are three conditions: a coherent source, a long baseline, and interference. For some unknown reason, nature was kind enough to provide us with all of the three necessary conditions. Because neutrinos interact so little, neutrinos maintain their coherence after propagation over long distances, coming from the Sun, cosmic rays, supernovae, accelerators, and reactors. These sources are naturally associated with rather long baselines. And most remarkably, significant interference effects require large mixing angles, which happened to be the case with neutrinos! Looking at it this way, it may not be a huge surprise that the neutrino oscillation was the first concrete evidence for physics beyond the Standard Model. In other words, the neutrino interferometry (a.k.a. neutrino oscillation) is a unique tool for studying physics at very high energies, already probing up to $\Lambda \approx 10^{14} \mathrm{GeV}$. Because of this line of argument, I'm a big fan of neutrino physics-so much so that I participated in the KamLAND experiment [17] spending some time building the detector, taking shifts, and serving on paper committees.

One sometimes hears the criticism that flavor physics experiments, quark, charged lepton, or neutrinos, have done little to improve our understanding of the underlying physics, compared to those experiments that focused on forces that led to the gauge theory and the Englert-Brout-Higgs mechanism. Indeed, we've known the pattern of quark masses and mixing angles already for some time, with no clear standard theory behind them.

We can, however, at least ask a question: does the patten of masses and mixings require a new structure or symmetries beyond what we know? I claim that we can answer this question.

I proposed the idea called anarchy, namely that the neutrino masses and mixings do not require any new symmetries to be understood, with Lawrence Hall and Neal Weiner [18. If there are no symmetries or quantum numbers that distinguish three generations of neutrinos, the neutrino mass matrix would consist of nearly random comparable numbers without a particular structure. We can understand their consequences basically by throwing dice. It actually leads to a definite prediction for the mixing angles: the probability distributions are given by the Haar measure of the compact groups, the unique measure that is invariant under both left- and right-translations [19]. Then the distributions in $x_{i j}=\sin ^{2} 2 \theta_{i j}$ turn out to be the same for all three angles, 


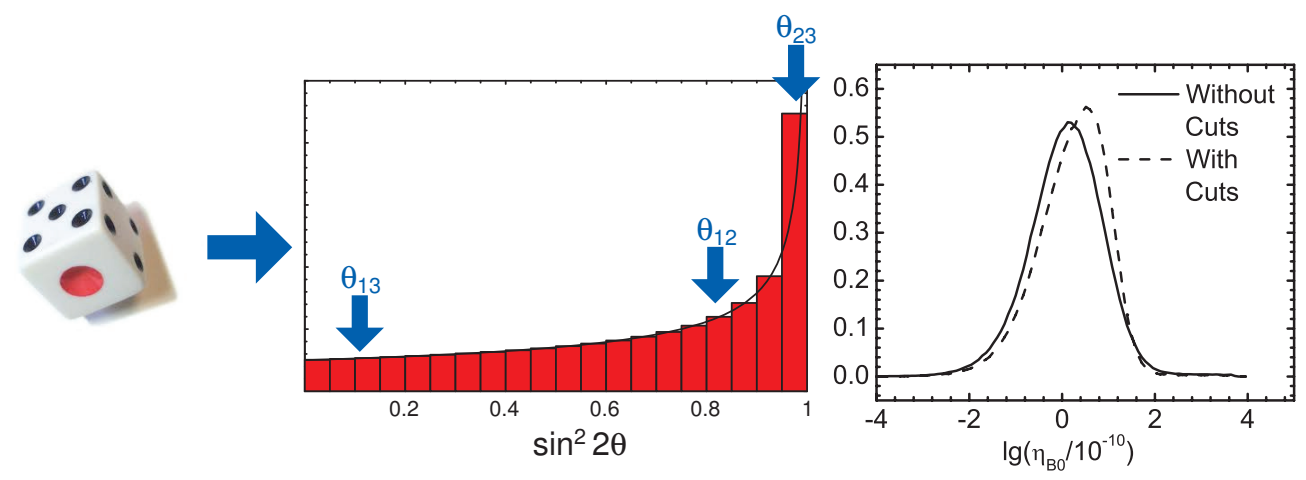

Figure 3. Left: Prediction of random matrices for the neutrino mixing angles. Right: consequence of anarchy on the baryon asymmetry of the Universe via the thermal leptogenesis scenario.

$P(x) d x=\frac{1}{2} \frac{d x}{\sqrt{1-x}}$, peaked at the maximal mixing $x=1$. Given this, it is not surprising that the neutrino oscillation was first discovered at the peak $x_{23} \approx 1$, then somewhere around the middle of the distribution $x_{12} \approx 0.86$, and finally somewhat down the tail $x_{13} \approx 0.09$ (Fig. 3 Left). In fact, André de Gouvêa and I did a Kolgomov-Smirnov test [20] to see if the one draw made by nature is consistent with this probably distribution, and found that it is $47 \%$ probable [21]! So we learned indeed that the neutrino masses and mixings do not require any deeper symmetries or new quantum numbers. On the other hand, quarks clearly do need additional input, which is yet to be understood.

The idea can be extended to the sector of right-handed neutrinos by assuming that they have a hierarchy akin to those in the charged leptons or quarks, $\epsilon^{2}: \epsilon: 1$. We take $\epsilon \approx 0.1$. With this structure, we can randomly generate the full left- and right-handed neutrino mass matrices. Xiaochuan Lu and I identified that the Gaussian measure is the unique choice based on a certain set of criteria, and found that the baryon asymmetry comes out extremely well (Fig. 3 Right) [22]. This is encouraging; in particular it is promising that the anarchy predicts that the distribution in the CP-violating effect would peak at $\sin \delta= \pm 1$ (or flat in $\delta$ ).

In fact, the $\mathrm{CP}$ violation in neutrino oscillation is the holy grail in neutrino experiments currently being planned and discussed. A possible CP violation (assuming no matter effect) is given in terms of a product of many factors,

$$
\begin{aligned}
P\left(\nu_{\mu} \rightarrow \nu_{e}\right)-P\left(\bar{\nu}_{\mu} \rightarrow \bar{\nu}_{e}\right)= & -16 s_{12} c_{12} s_{13} c_{13}^{2} s_{23} c_{23} \\
& \sin \delta \sin \frac{\Delta m_{12}^{2} L}{4 E} \sin \frac{\Delta m_{13}^{2} L}{4 E} \sin \frac{\Delta m_{23}^{2} L}{4 E} .
\end{aligned}
$$

It is remarkable that all factors are now found to be large enough to make this search feasible, the only unknown being the size of the CP violation $\sin \delta$ itself. Nature seems kind to us once again! It was also interesting to learn at this symposium that beyond the LBNE in the US and HyperK in Japan, there is a new discussion to use the European Spallation Source in Sweden with a much shorter baseline to look for CP violation [23]. Coming back to dark matter, there is a big ongoing effort on Weakly Interacting 
Massive Particle dark matter experiments from underground, cosmic, and accelerator experiments [24]. If the bet is right, we may see great discoveries sometime soon!

I argued that expeditions are needed to find where the next important energy scale is to solve the five mysteries. On the other hand, so-called energy frontier experiments, namely those that rely on high-energy colliders, target a rather specific energy scale. This leads us to ask the next question.

\section{Is the Energy Frontier Dead?}

The mantra in particle physics is to go to as high energy as technology (and money) allows. We are indeed going up a notch in 2015, restarting the LHC at 13-14 TeV. This is already exciting, improving reach on new particles by a factor of two. HighLuminosity LHC (HL-LHC) would further improve the reach by $20-30 \%$. We still have quite a bit of room for discoveries. More recently, there are discussions about a potential $100 \mathrm{TeV} p p$ collider with a much bigger tunnel around CERN.

However, I see a problem arguing for the next much higher energy machine now. Given that the discovery of the Higgs boson made the theory apparently complete, and the five mysteries I discussed have not yet set particular energy scales, I don't know how we can justify the energy of the next machine. Does this mean that there is no case we can make to build another high-energy collider? Is Energy Frontier dead?

It remains true that the best argument we have right now to expect new physics in the $\mathrm{TeV}$ range is the naturalness: we would like to avoid fine-tuning between the bare $m_{h}^{2}$ and the radiative correction (see, e.g., 25] for a plot). Even though many in the community are ditching the naturalness altogether, I still take the argument seriously because it has worked many times before.

One example I always bring up is the discovery of the positron [26, 27]. In classical electrodynamics, the Coulomb self-energy of the electron is linearly divergent, $\Delta m_{e} c^{2} \sim \frac{e^{2}}{r_{e}}$, where $r_{e}$ is the "size" of the electron. It would have required a fine cancellation between the "bare" mass of the electron (which must be negative by the way) and the correction to yield a small mass $m_{e} c^{2}=0.511 \mathrm{MeV}$. However, the discovery of the positron and quantum mechanics told us that the vacuum is always fluctuating, producing a pair of $e^{+} e^{-}$, that annihilates back to the vacuum within the time allowed by the uncertainty principle $\Delta t \sim \hbar / \Delta E=\hbar / 2 m_{e} c^{2}$. When you place an electron in this fluctuating vacuum, it may find a (virtual) positron near it and decide to annihilate it. Then the other electron that was originally in the vacuum fluctuation is now left out and becomes a "real" particle. It turns out that this process cancels the linear divergence exactly, leaving only a logarithmic divergence $\Delta m_{e} c^{2}=\frac{3 \alpha}{4 \pi} \log \frac{\hbar}{m_{e} c r_{e}}$. Even for an electron as small as the Planck distance, it amounts to only $9 \%$ correction. The cancellation is guaranteed by a (softly broken) chiral symmetry. You can see that the naturalness problem was solved by doubling the number of particles!

The idea of supersymmetry was pushed to repeat the history. Because the Higgs boson must repel itself, it also has a divergent self-repulsion energy $\Delta m_{H}^{2} \sim \lambda / r_{H}^{2}$. 
But by doubling the number of particles (namely introducing superpartners), there is a cancellation between the self-repulsion among Higgs bosons, and the induced attraction arising from the loop of higgsinos (fermionic partner of the Higgs boson). Again, the correction is down to a logarithmic divergence, $\Delta m_{H}^{2} \sim \frac{1}{(4 \pi)^{2}} m_{S U S Y}^{2} \log \left(\frac{\hbar}{m_{H} c r_{H}}\right)$.

In the case of the electron, new physics (positron) appears "early" at the Compton wave length $\hbar / m_{e} c \approx 400 \mathrm{fm}$ well before we get down to the smaller "classical radius of electron" $r_{c}=e^{2} / m_{e} c^{2} \approx 1 \mathrm{fm}$ where the theory becomes fine-tuned. In another well-known case, however, nature did fine-tune it so that the discovery was delayed.

The example is COBE (Cosmic Background Explorer) [28] that discovered the CMB anisotropy. People expected anisotropy at the level of $10^{-5}$ so that the observed largescale structure can be explained. But the search went on, so much so that people started writing articles questioning the inflationary cosmology itself. When COBE discovered the quadrupole moment, it was small. Actually, compared to our best prediction today based on the WMAP data, it was nearly an order of magnitude smaller than theory. This is usually understood today as a consequence of cosmic variance, namely that the quadrupole moment has only $2 l+1=5$ numbers to be measured and hence is subject to a statistical uncertainty of $O(1 / \sqrt{5})$. I find the observed quadrupole moment to be fine-tuned at the $2 \%$ level.

Note that the inflation was invented to solve the naturalness problems, horizon problem and flatness problem of the standard Big Bang cosmology. It worked: the current data beautifully confirm predictions of inflation. But it was a little fine-tuned and it required patience and more work.

So the moral I draw from these examples is that the naturalness argument generally does work. But there are cases where fine-tuning at the level of a few percent or even few per-mille (some examples in nuclear physics are well-known, see [29]). Looking back at Fig. 1, we have not fully explored down to that level of not-that-fine-tuning yet. And it took ten years for Tevatron to discover top. Patience pays, hence my optimism.

But it is true that it is a slippery slope how much fine tuning we tolerate. Percent? Per-mille? $10^{-6}$ ? It is quite subjective or matter of taste, and we cannot firmly set the energy for the next collider based on this argument with any confidence. Back to the question again: is there any justification for the next high-energy collider?

I believe there is. The Higgs boson is the only newly discovered particle at the LHC so far, but it is clearly an unusual particle. And we know what energy is required to study it already, because we know its mass. We know where to look.

What is unusual about the particle discovered? To the extent ATLAS and CMS have managed to study it so far, it is consistent with $J^{P C}=0^{++}$. It has no spin! We have never seen an elementary spinless particle before, so this looks like a new breed. Matter particles like quarks and leptons come in three generations, and we seem to understand their context. That is, they look familiar, they are siblings, and are a part of a big family. Same with the force particles. They all belong to some kind of gauge group. Even though the groups differ, they follow the same principle. Again, they have relatives, and have context. But the Higgs boson is totally different. It is one of its 
kind, with no context within the Standard Model. That means that we introduced a kind of particle nobody has seen before. It is a faceless (spinless) intruder to our happy family. Yet it is supposed to do the most important job in the theory. The whole idea looks so artificial!

In fact, I couldn't believe the artificial aspect of the Standard Model at all when I studied it back in grad school, so I became one of the guilty ones to propose the Higgsless theories [30]. But this idea now appears dead.

Facing its existence, we are still puzzled by another question. The closest analogy we have in familiar systems is the superconductivity we can study in the laboratory. Instead of giving mass to $W$ and $Z$-bosons, superconductivity gives mass to the photon. In other words, due to the Meissner effect, the magnetic field is repelled by the superconductor, allowing it to enter only by a finite distance (the penetration depth). The magnetic field is short-ranged inside the superconductor! This case, however, is well understood. It is caused by the instability of Fermi surface when electrons are attracted to each other by a weak force from the phonon exchange. Cooper pairs condense, making the magnetism short-ranged. On the other hand, the Standard Model does not tell us why the Higgs boson condenses in our Universe. This is not only artificial, it is unsatisfying.

There are ideas to give context to the spinless Higgs boson. There may be many siblings and relatives. The Higgs boson is just one among the big spinless tribe, ome which happens to condense because of an attractive force induced by the top-quark loops. This idea is known as supersymmetry. An additional Higgs doublet is its sibling, and there are many other spinless squarks and sleptons, that are its relatives. On the other hand, it may be composite, just like spinless pions are made of spin 1/2 quarks. In this case a new dynamics would be required to bind the constituents together. Or the Higgs boson may actually be spinning, but if it does in extra dimensions we cannot see, so we perceive it to be spinless. In such a case, the Higgs boson may actually be a gauge boson or even graviton. These are all familiar ideas we discussed for solving the naturalness problem. Here I'm not using the naturalness argument at all; but I still come back to a similar set of ideas, namely that there are good reasons to continue discussing these ideas.

Then, what should we do? Of course, we should study this intruder as much as we can! If we look closely enough, maybe we can tell it it has siblings or relatives. We may find it has a finite size. Or we may bring it back to spin in our dimensions.

Fortunately, the observed mass of $125 \mathrm{GeV}$ is the best case scenario. It allows us to measure branching fractions to $b \bar{b}, W W^{*}, Z Z^{*}, g g, \tau^{+} \tau^{-}, c \bar{c}, \gamma \gamma, Z \gamma$, possibly even $\mu^{+} \mu^{-}$. Some of them would not be accessible if the Higgs were lighter or heavier by just a few tens of $\mathrm{GeV}$. It is actually a dream case for experiments!

Looking back at the history of collider experiments, precision measurements using leptons often revealed the next energy scale; we went up there with hadrons, and we indeed found new things, which we further studied with lepton probes. One full cycle is the precision measurements of neutral currents in polarized electron deuteron scattering at SLAC. The measured $\sin ^{2} \theta_{W}$ predicted the masses of $W$ and $Z$. Spp̄S was built to 
discover them, which indeed did. After that LEP was built to study them precisely and we nailed the gauge sector of the Standard Model.

The next cycle starts with LEP predicting the top quark and Higgs boson masses. Tevatron and LHC were built for this purpose, and as we know, thy did discover the predicted particles. The obvious thing to do next is to study them precisely to nail the top and Higgs sector at another lepton machine.

If the history is any guide, the future precision measurement of the top and Higgs sector would tell us the next energy scale we should go after. We are on a scavenger hunt. The Higgs boson discovered is a lamp post, we need to look carefully at what's under it, and hope to find a clue to the next destination.

Another reason why the precision study of the Higgs boson is exciting is that the Higgs boson may be a portal to a new sector outside the Standard Model. It may, for example, be a sector of the dark matter particle. To probe an operator $\mathcal{O}$ (with mass dimension $d$ ) in the new sector, we need its coupling to the Standard Model particles. As we discussed before, all operators in the Standard Model are of dimension four, except for the Higgs mass-squared. Therefore the couping is suppressed as e.g., $\frac{1}{\Lambda_{U V}^{d}} \mathcal{O} F_{\mu \nu}^{2}$, while the coupling to the Higgs goes as $\frac{1}{\Lambda_{U V}^{d-2}} \mathcal{O} H^{\dagger} H$. Thus the coupling to the Higgs is enhanced by $\Lambda_{U V}^{2}$ relative to other operators. The Higgs boson may be the window to the new world.

In addition, once we build a new lepton collider to study Higgs and top precisely, we can still hope that it discovers new particles directly. It is not true that LHC excluded everything below TeV. Even a slepton of, say, $150 \mathrm{GeV}$ is still allowed if it decays into a neutralino heavier than $80 \mathrm{GeV}$ or so. LHC will improve limits to heavier sleptons, but not much to close the gap when their masses are close.

Given this, I'd think the strategy is clear. We start with what we have got. We build a lepton collider that can study the top quark and Higgs boson precisely. This will be an evolutionary program, starting with the $Z h$ threshold, measuring branching fractions and couplings to $Z, W, b, c, \tau, g, \gamma$, even the decay into invisible particles. Then on to the $t \bar{t}$ threshold to study the top quark compositeness, say, going up further in energies to make use of new processes such as $W W$-fusion, $t \bar{t} h$ production for $y_{t t h}$, and multiple Higgs production for $\lambda_{h h h}$. But we should keep our eyes open to the possibility that we may also discover new particles along the way. Just in case we obtain a new piece of information on new particles from the LHC, the lepton collider should be extendable. If we do see new particles, we should have the capability of studying them in model-independent way, and to determine their quantum numbers, spins, masses, and couplings. The machine should be one that we know how to build, so that we can propose it as soon as an opportunity presents itself.

The planned International Linear Collider (ILC) fits this bill very nicely, and its scientific case was judged very strong in the European Strategy document adopted by CERN Council in May [31]. The technology required for this is mature, thanks to the Global Design Effort (GDE) led by Barry Barish that finished the Technical Design 


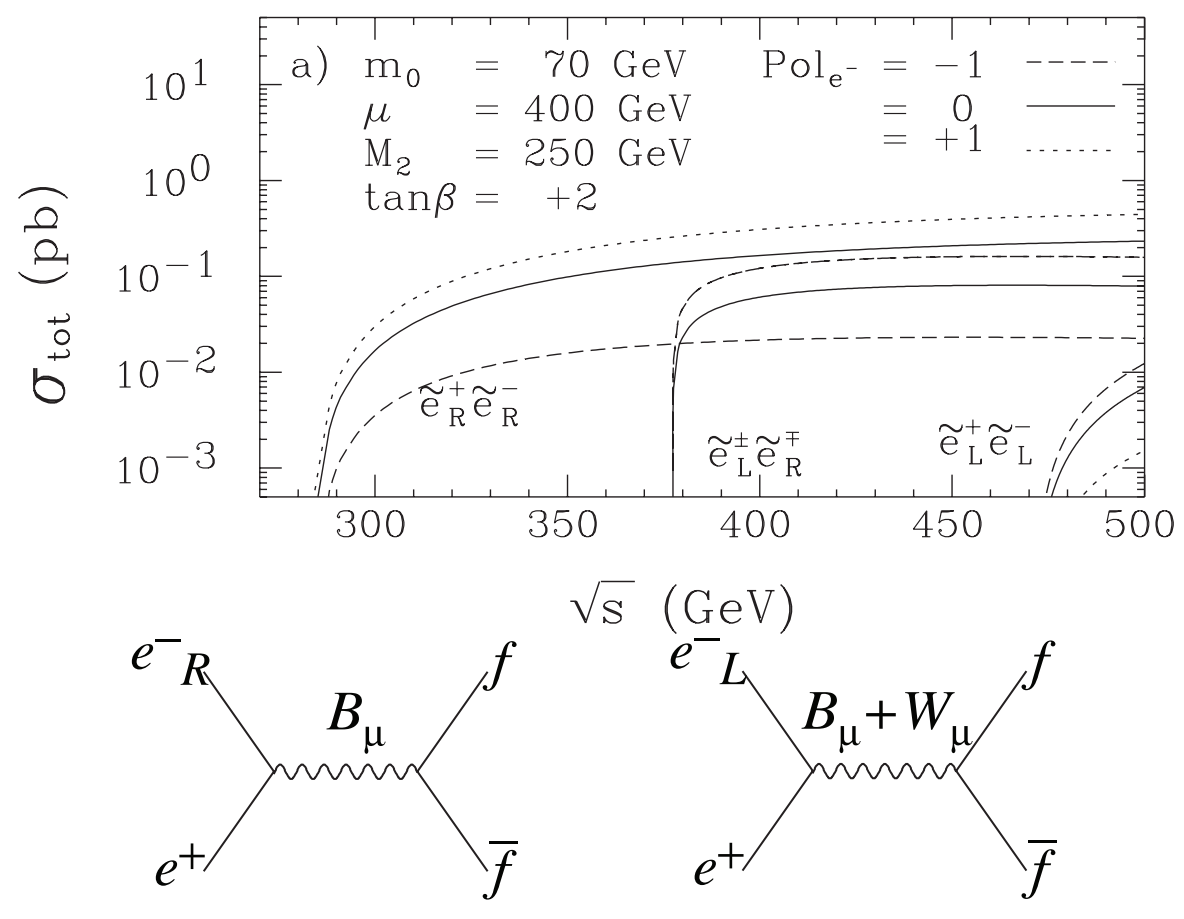

Figure 4. Top: The cross sections for producing pairs of right-handed slectron vs lefthanded selectron for various beam polarizations [34]. Bottom: $s$-channcel production processes for new particles with polarized electrons.

Report (TDR) this year [32]. It is extendable, so that we can increase the energy if needed and affordable. In addition, the longitudinal beam polarization provides a crucial tool. The bottom diagrams in Fig. 4 show that different electron polarization has different gauge bosons in the $s$-channel. At these energies, we can neglect $m_{Z}^{2} \ll s$ as an approximation. Then the gauge bosons exchanged are either $U(1)_{Y}$ gauge boson $B_{\mu}$, or the neutral $S U(2)_{L}$ gauge boson $W_{\mu}^{0}$. The right-handed electron is a singlet under $S U(2)_{L}$ (the subscript $L$ stands for left-handed), and does not couple to $W_{\mu}^{0}$. Therefore, the $s$-channel production goes as $\left|g^{\prime} Y_{f}\right|^{2}$ and directly measures the hypercharge $Y_{f}$ of the new particle $f$. On the other hand, the left-handed electron couples to both, and the cross section goes as $\left|g^{\prime} Y_{f}+g I_{3 f}\right|^{2}$. Knowing $Y_{f}$, we can determine $I_{3 f}$ modelindependently. One can see how much the cross sections vary depending on the beam polarization in the top plot of Fig. 4 .

There are many studies of spin and mass measurements, which can be done even when particles decay into invisible particles. Combination of the LHC and ILC data may allow us to even compute the cosmological abundance of the invisible particle, possibly verifying that it is the dark matter of the Universe [33].

But isn't ILC too expensive to be ever built? Through some miracle, many politicians in Japan are interested in hosting the ILC as a global project. They would like to open up the country to talented, intellectual people from abroad. They would like to find prestige in hosting highly visible large international project. They also want to use the ILC to build up infrastructure, a technological base, and they hope to 
find economic benefits. More than $20 \%$ of the Diet members signed up to support the ILC, in a group named Federation of Diet Members for Promotion of the ILC. When Lyn Evans visited Japan in March, the prime minister Shinzo Abe agreed to meet him [35], and he said that he appreciated the significance of the ILC as "a dream for humankind." His opening address in the 183rd session of the Diet mentioned advanced accelerator technology as one of the innovation areas in which he wants Japan to excel [36]. There are many industry associations actively supporting the ILC; the media is highly interested as well. And the discovery of the Higgs boson has fueled interest even further. I'd think there is a high enough level of interest for the Japanese government to initiate discussions with other potentially interested countries to form an international framework for a global ILC project hosted in Japan. I am not absolutely sure; but it doesn't look impossible so far.

Having discussed expeditions to high-energy scales, and precision studis of the Higgs and top to identify the next energy scale(s), there are plenty of things that we can and will do in the near future in our field. However, it still leaves one question that has been haunting me.

\section{Do we ever understand Dark Energy?}

Dark Energy is such a big mystery that I cannot gauge how we may ever understand it. Does this mean that it is useless to try to measure its properties precisely?

I don't know. But all I can say is that a percent-level or better measurement is what I consider precise. If the equation of state parameter $w \equiv p / \rho=0 \pm 0.01$, I may give up and say it is the cosmological constant, accidentally small in a landscape of $10^{500}$ universes. But it may turn out to be $w=0.05 \pm 0.01$, pointing us in a new direction. I believe that it is worth the try.

I lead a major dark energy experiment called SuMIRe (Subaru Measurement of Images and Redshifts) [37]. It combines imaging and spectroscopy on the 8.2m-diameter Subaru telescope, a major step up from the wildly successful SDSS (Sloan Digital Sky Survey) [38]. The first stage is the approved imaging survey with Hyper Suprime-Cam for 300 nights, with nearly 0.9 billion pixels with a field of view of 1.7 square degrees. It will image hundreds of millions of galaxies. The next stage is a spectroscopic survey with the Prime Focus Spectrograph for (hopefully) 300 nights, with 2400 optical fibers, controlled robotically, being targeted at galaxies chosen from the imaging survey. For instance, it will yield a model-independent measurement of the evolution of the dark energy fractions as a function of the redshift (left Fig. 5) and provide a test of general relativity at cosmological distances (right Fig. 5).

We should do what we can do, and we will see what we find! 

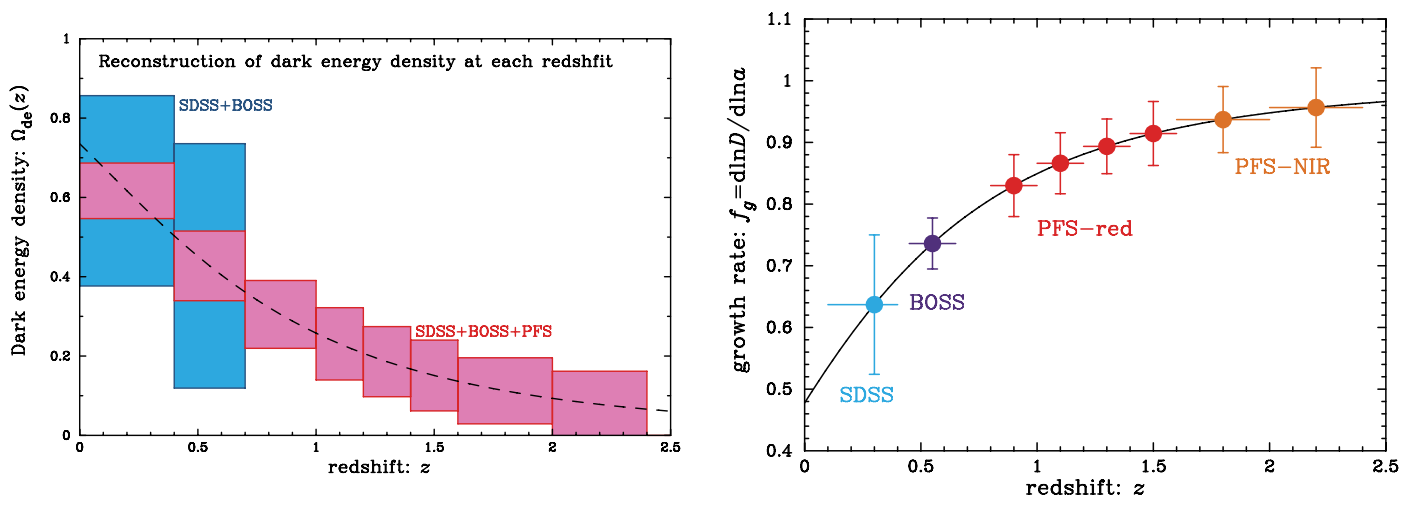

Figure 5. Left: Projected accuracy in measuring $\Omega_{D E}(z)$ using PFS on Subaru telescope. Right: Test of general relativity on cosmological distances using the growth rate of structure. Both taken from 39 .

\section{Appendix A. Pushing back on fine-tuning}

Take supersymmetry. There are two issues facing the experimental data. The first one is that the mass of the discovered Higgs boson $m_{h}$ is a little too high within the MSSM, which predicts $m_{h} \leq m_{Z}$ at the tree-level. Even though the Higgs mass can be pushed up by the radiative correction as

$$
m_{h}^{2} \simeq m_{Z}^{2}+\frac{3}{4 \pi} h_{t}^{4} v^{2} \log \frac{m_{\tilde{t}_{1}} m_{\tilde{t}_{2}}}{m_{t}^{2}},
$$

it would require a large scalar top mass, which would feed into the radiative correction to the Higgs mass-squared term

$$
\Delta m_{H_{u}}^{2} \simeq-12 \frac{h_{t}^{2}}{16 \pi^{2}} m_{\tilde{t}}^{2} \log \frac{\Lambda_{U V}}{\mu_{I R}} .
$$

Therefore, a larger physical Higgs mass in the MSSM indirectly implies exponentially worse fine-tuning in $m_{H_{u}}^{2}$ between the bare parameter in the Lagrangian and the radiative correction above.

This can be avoided if there is an additional contribution to the Higgs self-coupling, such as in the massive NMSSM $W=\lambda S H_{u} H_{d}+\frac{1}{2} M S^{2}$. However the contribution decouples in the limit $M \gg m_{h}$ as

$$
\lambda^{2}=1-\frac{M^{2}}{M^{2}+m_{S}^{2}} .
$$

This can be prevented for a large soft mass $m_{S}^{2} \gg M^{2}$ as a non-decoupling effect [40], but $m_{S}^{2}$ then feeds into $\Delta m_{H_{u}}^{2}=\frac{1}{16 \pi^{2}} 2 \lambda^{2} m_{S}^{2} \log \frac{\Lambda_{U V}}{\mu_{I R}}$, re-introducing the fine-tuning.

Together with Xiaochuan Lu, Josh Ruderman, and Kohsaku Tobioka, we've come up with an idea that we call semi-soft supersymmetry breaking [41]. Using the NMSSM (Next-to-Minimal Supersymmetric Standard Model) of the Dirac-type, $W=\lambda S H_{u} H_{d}+M S \bar{S}$, the singlet field $\bar{S}$ couples to the rest of the model only through a dimensionful coupling $M$. It can then be proven that the limit $m_{\bar{S}}^{2} \rightarrow \infty$ 


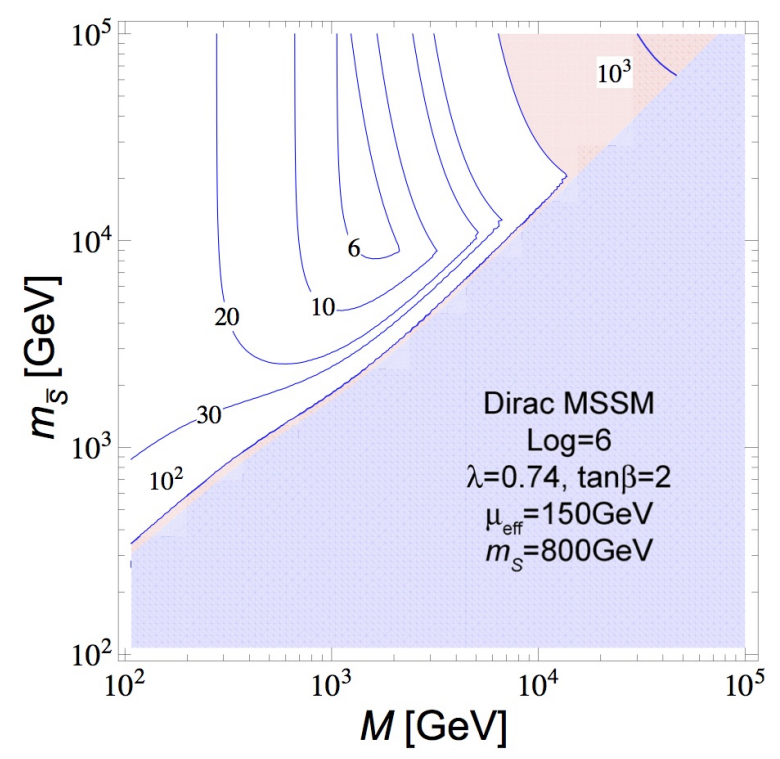

Figure A1. Degree of fine-tuning in Dirac NMSSM that shows it can be as good as a factor of six even when $m_{\bar{S}} \gg m_{h}$. Taken from [41].

does not re-introduce fine-tuning (Fig. A1) even though it looks like a hard breaking of supersymmetry, hence semi-soft.

The second problem with supersymmetry is its non-observation in direct searches. It is well-known that a quasi-degenerate spectrum among supersymmetric particles makes the search difficult because of small $Q$-values in decays and hence small $E_{T}$ (see, e.g., [42]). However, such a spectrum lacked theoretical motivation: in particular, why should scalars and gauginos be degenerate?

Together with Yasunori Nomura, Satoshi Shirai, Kohsaku Tobioka, I proposed that supersymmetry broken by boundary conditions in extra dimensions would automatically give the same mass to all gauginos and sfermions at the tree-level split only by loop effects [43], similar to the Universal Extra Dimension (UED) [44]. Correspondingly, the experimental limit is weaker. A dedicated search with ISR should improve the limit like in the UED case [45].

This work was supported in part by the U.S. DOE under Contract No. DEAC0376SF00098, by the NSF under Grant No. PHY-1002399, by the JSPS Grant (C) No. 23540289, by the FIRST program Subaru Measurements of Images and Redshifts (SuMIRe), CSTP, and by WPI, MEXT, Japan.

\section{References}

[1] G. Aad et al. [ATLAS Collaboration], Phys. Lett. B 716, 1 (2012).

[2] S. Chatrchyan et al. [CMS Collaboration], Phys. Lett. B 716, 30 (2012).

[3] P. A. R. Ade et al. [Planck Collaboration], arXiv:1303.5082 [astro-ph.CO].

[4] C. Afonso et al. [EROS Collaboration], Astron. Astrophys. 400, 951 (2003). 
[5] D. N. Spergel et al. [WMAP Collaboration], Astrophys. J. Suppl. 148, 175 (2003).

[6] W. Hu, R. Barkana and A. Gruzinov, Phys. Rev. Lett. 85, 1158 (2000).

[7] Y. Fukuda et al. [Super-Kamiokande Collaboration], Phys. Rev. Lett. 81, 1562 (1998).

[8] Q. R. Ahmad et al. [SNO Collaboration], Phys. Rev. Lett. 89, 011301 (2002).

[9] K. Eguchi et al. [KamLAND Collaboration], Phys. Rev. Lett. 90, 021802 (2003).

[10] F. P. An et al. [DAYA-BAY Collaboration], Phys. Rev. Lett. 108, 171803 (2012).

[11] S. Perlmutter et al. [Supernova Cosmology Project Collaboration], Astrophys. J. 517, 565 (1999).

[12] A. G. Riess et al. [Supernova Search Team Collaboration], Astron. J. 116, 1009 (1998).

[13] K. Abe et al. [Belle Collaboration], Phys. Rev. Lett. 87, 091802 (2001).

[14] B. Aubert et al. [BaBar Collaboration], Phys. Rev. Lett. 87, 091801 (2001).

[15] C. Jarlskog, Phys. Rev. Lett. 55, 1039 (1985).

[16] I thank Zoltan Ligeti for the concept for this schematic plot.

[17] Kamioka Liquid scintillator Anti-Neutrino Detector http://www.awa.tohoku.ac.jp/kamlande/

[18] L. J. Hall, H. Murayama and N. Weiner, Phys. Rev. Lett. 84, 2572 (2000).

[19] N. Haba and H. Murayama, Phys. Rev. D 63, 053010 (2001).

[20] A. de Gouvêa and H. Murayama, Phys. Lett. B 573, 94 (2003).

[21] A. de Gouvêa and H. Murayama, arXiv:1204.1249 [hep-ph].

[22] Xiaochuan Lu and Hitoshi Murayama, in preparation.

[23] E. Baussan et al. [ESSnuSB Collaboration], arXiv:1309.7022 [hep-ex].

[24] René Ong, in this proceedings.

[25] C. F. Kolda and H. Murayama, JHEP 0007, 035 (2000).

[26] H. Murayama, hep-ph/9410285.

[27] H. Murayama, hep-ph/0002232.

[28] Cosmic Background Explorer http://lambda.gsfc.nasa.gov/product/cobe/

[29] Nima Arkani-Hamed in this proceedings.

[30] C. Csaki, C. Grojean, H. Murayama, L. Pilo and J. Terning, Phys. Rev. D 69, 055006 (2004) hep-ph/0305237.

[31] http://council.web.cern.ch/council/en/EuropeanStrategy/ESParticlePhysics.html

[32] http://www.linearcollider.org/ILC/Publications/Technical-Design-Report

[33] E. A. Baltz, M. Battaglia, M. E. Peskin and T. Wizansky, Phys. Rev. D 74, 103521 (2006).

[34] T. Tsukamoto, K. Fujii, H. Murayama, M. Yamaguchi and Y. Okada, Phys. Rev. D 51, 3153 (1995).

[35] http://www.kantei.go.jp/jp/96_abe/actions/201303/27ilc_hyokei.html

[36] http://www.kantei.go.jp/jp/96_abe/statement2/20130228siseuhousin.html

[37] Subaru Measurements of Images and Redshifts http://sumire.ipmu.jp/en/

[38] Sloan Digital Sky Survey http://www.sdss.org

[39] R. Ellis et al. [PFS Team Collaboration], arXiv:1206.0737 [astro-ph.CO].

[40] M. Dine, N. Seiberg and S. Thomas, Phys. Rev. D 76, 095004 (2007).

[41] X. Lu, H. Murayama, J. T. Ruderman and K. Tobioka, arXiv:1308.0792 [hep-ph].

[42] T. J. LeCompte and S. P. Martin, Phys. Rev. D 85, 035023 (2012).

[43] H. Murayama, Y. Nomura, S. Shirai and K. Tobioka, Phys. Rev. D 86, 115014 (2012).

[44] H. -C. Cheng, K. T. Matchev and M. Schmaltz, Phys. Rev. D 66, 056006 (2002).

[45] H. Murayama, M. M. Nojiri and K. Tobioka, Phys. Rev. D 84, 094015 (2011). 